\title{
Study on the Evolution Mechanism of Public Crisis of Sudden Animal Epidemics
}

\author{
Li Yanling ${ }^{1}$ Wang Wei $^{1} \quad$ Liu Bing $^{1}$ Zhang Xie ${ }^{1}$ \\ ${ }^{1}$ College of Humanities and Social Science, Hunan Agricultural University, Changsha, P. R. \\ China 410128
}

(liyanling1964@163.com,violet_wwei@163.com,535864262@qq.com, zhangxie422@126.com)

\begin{abstract}
With the application of the analytical tools of staged evolution mechanism, the paper implements a stage-based research on the public crisis of sudden animal epidemics based on time sequence and specific life cycles. The paper, by presenting a stage-based mechanism analytical model with "3 stages, 3 ups-and-downs and 5 key points", maintains that the public crisis of sudden animal epidemics is developed and influenced by the integrated function of natural evolution and manual emergency intervention, and the manual emergency intervention plays a critical role in the epidemic developing tendencies.
\end{abstract}

KeyWords: Public Crisis, Evolution Mechanism, animal epidemics

\section{Introduction}

The public crisis evolution refers to the integrated process of natural evolution and manual emergency intervention [1]. And evolution mechanism indicates the development rule of a sudden public crisis, including its occurrence, development and disappearance. [2] At present, the research for various analytical types on evolution mechanism of sudden public crisis can be divided as stage-based type, diffused type, cause-and-effect type and scene type. Among all of these, the staged-based type is successfully achieved in research with fruitful research findings. For instance, three-stages theory includes the model of "Pre-crisis, Crises and Post-crisis" which is highly appreciated by Birch and Cuth ; four-stages theory embodies Robert Heath's 4R model (2001) and Fink's F model (1986); five-stages theory contains
Mitroff's M model (1994); and seven-stages theory involves Tumer's model of risk-phase (1976) and Ibrahim Razi's risk model (2002, 2003). However, the study on the evolution mechanism of public crisis of sudden animal epidemics basically maintains in a scattered, qualitative and descriptive state [3]. In terms of prevention and control mechanism construction, some scholars' researches start with the relationship between the prevention and control of epidemics and the development of animal husbandry, and point out that a rounded and profound study in analytical model for evolution mechanism of public crisis of sudden animal epidemics is recommended [4]. These researches comprehensively describe the current situations, epidemical tendencies and developing rules of swine diseases in China, although lacking of a more direct development mechanism study on how the epidemics turns to be public crises.

\section{Model}

The research of Merle Jacob and Tomas Hellstrom (2000) indicates that under the circumstances of unconventional management, the spread and interaction of secondary and derivative disasters exist in the developing mechanism of public crisis, which is much more complicated compared with the situation of conventional emergencies. Rooted on pre-researches, this paper constructs a staged mechanism analytical model of "3 stages, 3 ups-and-downs and 5 key points" for public crisis of sudden animal epidemics with the application of staged evolution analytical tools and the integration of the advantages of various analytical models. (Figure 1) 


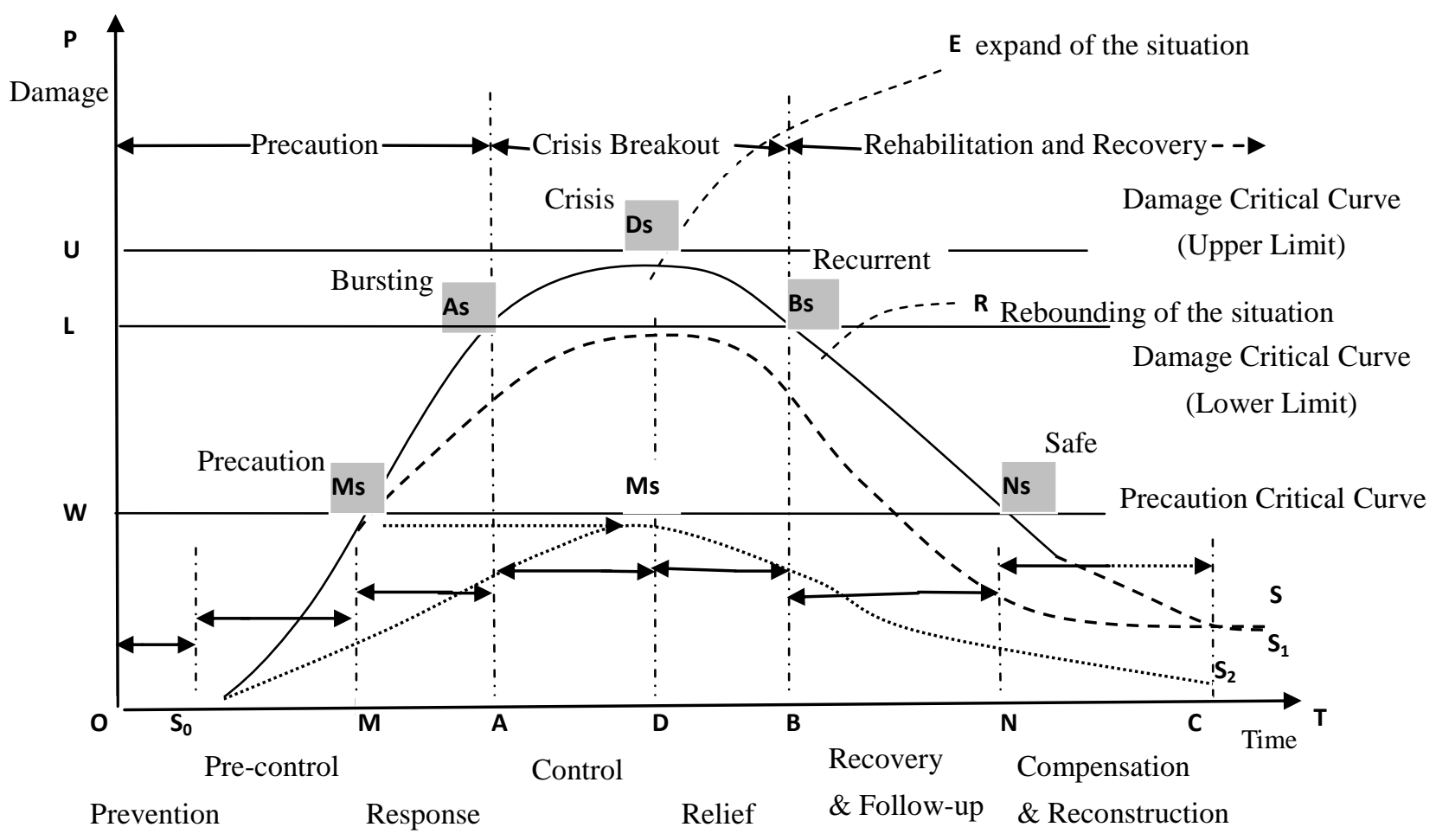

Fig. 1: Model of Staged Evaluation Mechanism of Public Crisis of Sudden Animal Epidemics

Stage-based evaluation mechanism researches mainly follow the time sequence and specific life cycle theory to study stages of public crisis events of sudden animal epidemic. Stage-based study should establish all kinds of emergency Stage-based model, and reveal the main features, propose different emergency measures according to the different stages. The model of staged evaluation mechanism of public crisis of sudden animal epidemics is corresponding with the model of real damage evolution of sudden animal epidemics (Figure $2)$; it reflects the essential evolution nature law of public crisis of sudden animal epidemic damage with the coactions of "natural evolution and manual emergency intervention". In Figure 1, the OP curve shows the animal epidemic actual damage degree (volume), the OT curve is the time axes. According to the curve change of epidemic actual damage, the public crisis of sudden animal epidemic could be divided into 3 different stages: the precaution stage (OA), crisis breakout stage (AB) and rehabilitation and recovery stage(BC).There are three curves in the figure 1 which are called the "3 ups-and-downs" to show the actual damage in the public crisis of sudden animal epidemic. The solid curve "SOS" is the evolution curve of the public crisis of sudden animal epidemic. There are 5 key points on the curve: the "Ms" point (the time point goes across the $\mathrm{M}$ point on the OT curve) is the crossing point of the epidemic damage curve SOS and "W" curve (Precaution Critical Curve), we call the "Ms" point as the precaution point. At this time, all kinds of crisis characterization alarm that the epidemic is on the phase of before-crisis stage; with the development of epidemic situation, the SOS curve quicken up rises and goes across with the "L" curve (Damage Critical Curve ,Lower Limit) on the point "As" (the time point goes on the A point of OT curve), we call the "As" point as the bursting point. At this time all kinds of crisis characterization proliferate suddenly and massively, the public crisis breaks out across-the-board and the damage rapidly blow up; In Interference of different benefit entity, and because of the attenuation of the proliferative capability of sudden animal epidemic, the proliferative speed of epidemic damage is controlled, the SOS curve raises is slowed and goes across with the upper limit curve of social damage critical curve (U, Upper Limit) at the "Ds" point (the time point goes on the D point on of OT curve). We call the "Ds" point as the crisis or "Disaster" point. As a result of effective public crisis 
management of sudden animal epidemic, the SOS curve could turn to the "good" direction when it approaches the "Ds" point (the Ds point become the actual turning point). After that the SOS curve could goes down across the social economic damage curve (L curve), and now the situation is at the rehabilitation and recovery stage. When the SOS curve goes across with the L curve at the "Bs" point (the time point goes on the B point of the OT curve), we call the "Bs" point as the recurrent point. From this time on, the public crisis of sudden animal epidemic start goes back to the normal situation; when the SOS goes down rapidly and goes across with the early warning curve (precaution critical curve, W) at the "Ns" point (the time goes at the $\mathrm{N}$ point of the OT curve), we call the "Ns" point as the safe point. When the curve goes under the "Ns" point, the public crisis of sudden animal epidemic damage is at the situation of normal management, and the SOS curve gradually restores horizontal movement and goes into a new life cycle of public crisis of sudden animal epidemic.

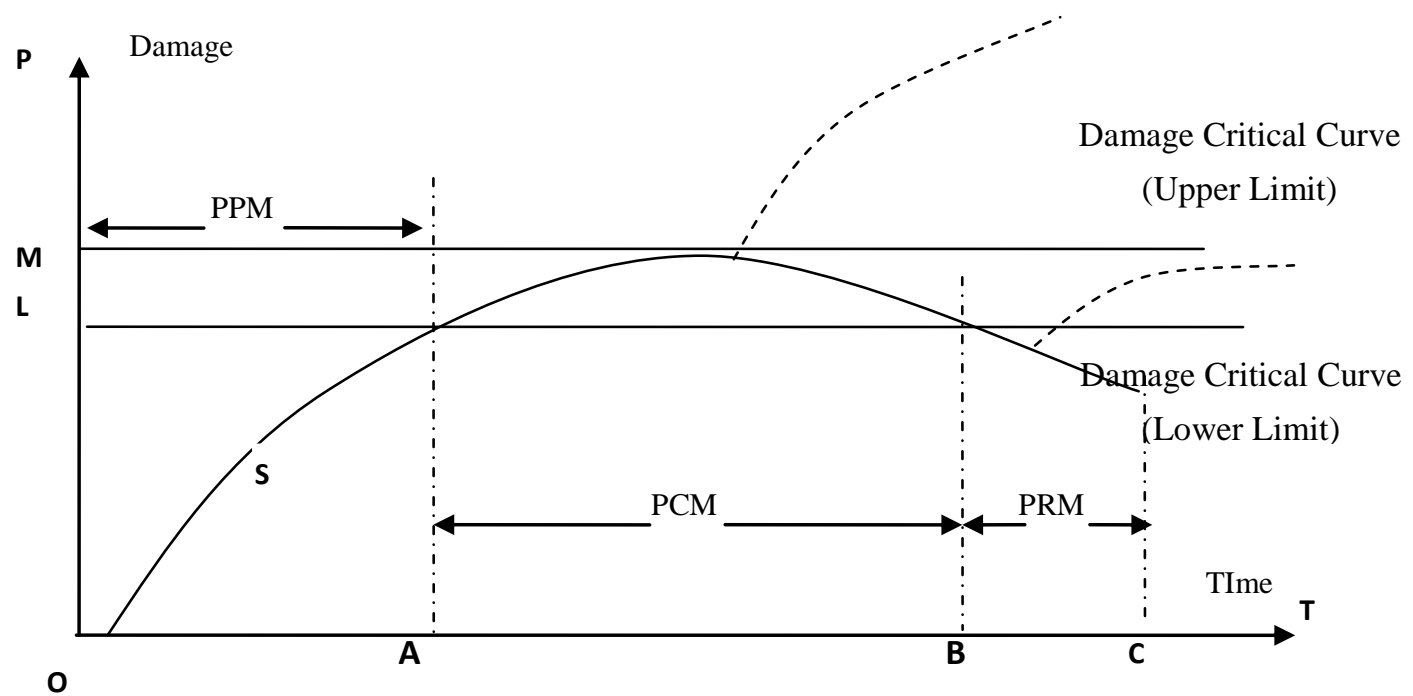

PPM( Phase of Precaution Management),

PCM(Phase of Crisis Management),

PRM(Phase of Rehabilitation Management)

Fig 2: Mode of Real Damage Evolution of Sudden Animal Epidemics

\section{Point of view}

We should pay more attention to three points in this process. Firstly, after sudden animal epidemic occurred, the location of the inflexion on the SOS curve depends on the measures of the precaution management, such as prevention, pre-control, response and so on, and if handled improperly, the epidemic of the damage curve will break through the upper limit of damage critical curve (U, Upper limit), so that the worsening of the situation and a larger public crisis break out, as shown in dotted curve E (the expand of the situation).

Secondly, as a result of scientific contingency plans, adequate emergency preparedness and effective crisis management, the damage curve of sudden animal epidemic can be more flat, even its highest point do not break through the As points, as shown in Figure 1, semi-shown in dotted curve $\mathrm{S}_{0} \mathrm{~S}_{1}$. The best effect is the damage of epidemic controlled under the precaution critical curve, and this time, the warning point moved to the time point $\mathrm{D}$, and the damage curve of sudden animal epidemic is almost smooth as dotted curve $\mathrm{S}_{0} \mathrm{~S}_{2}$ in Figure 1 (SOS, SOS ${ }_{1}$ and $\mathrm{SOS}_{2}$ constitute the "3 ups-and-downs "). However, if managed improperly, the damage of epidemic is possible to be reversed, and could goes up to the $\mathrm{L}$ curve to return to a state of crisis after the $\mathrm{S}_{0} \mathrm{~S}_{1}$ curve goes down at the recurrent point, as shown in the dotted curve 
$\mathrm{R}$ ( Rebounding of the situation ).

Thirdly, the lowest point which restored to the horizontal movement at the damage curve of sudden animal epidemic (SOS, SOS 1 and $\mathrm{S} \mathrm{S}_{2}$ ) is always higher than the minimum damage point ( $\mathrm{S} 0$ point) at the initial stage of the sudden animal epidemic, which means that in every event of sudden animal epidemic would have a certain socio-economic losses, and when a similar sudden incident occurs in the future, the extent of the damage (the scale or level) will rise to a higher degree. In the process of sudden animal epidemic including appearance, development and elimination, the actual damages from the initial point $\mathrm{O}$ to the situation of normal management-the end point C-experienced three major stages including precaution stages, crisis breakout stages, rehabilitation and recovery stage. In the whole process it can also be divided into six sub-stages including prevention, pre-control, response, control, relief, recovery \& follow-up, compensation \& reconstruction. Actual damage from small to big, reaching the maximum crisis point and then downwardly from big to small return to normal management. This is a complete evolutive rule of public crisis of sudden animal epidemic, called as the evaluation mechanism.

\section{Conclution}

From above analyses, manual emergency management does influence the direction of epidemic changes. The behavior decision model of emergency interferences from farmers, social groups and local governments has an important effect on the occurrence, development, diffusion, derivation and amplification of damage of sudden animal epidemics_ a kind of pubic crisis under unconventional state. However, a further empirical study is needed on how and to what extent does the influence affect the epidemics changes. The most critical part for the management and interference lies in the study of five key points (precaution point, bursting point, crisis point, recurrent point, safe point ) in development rules, i.e. to define three critical lines. It is suggested to take the animal number of deaths from diseases within a certain period of time or the resource quantity mobilized by public sector in response to the epidemics as the reference standard for the definition of the critical lines, but a further empirical analysis is required to complete the research as well.

\section{References}

[1]Fang Zhigen, Yang Baohu, Lu Zhipeng, Liu Sifeng, Chen Ye, Chen Wei, Yao Guozhang. The GERT Network Model Study of Disaster Evolution Based on Bayes Inference[J]. China Management Science, 2009, (17) 2: 102-107.(In Chinese)

[2]Qi Mingliang, Chi Hong, Zhao Hong, Sun Yin. A Research Review on the Public Emergency Management[J], Public Management and Public Policy, 2006, (18) 4:35-45.(In Chinese)

[3]Yang Jing, Chen Jianming, Zhao Hong. The Classification of Emergency in Incident Management[J]. Management Review, 2005, (17)4:37-42.(In Chinese)

[4]Ibrahim M Shaluf, Fakllarul-razi Ahmadun , Sa'ari MnstaPha. Technological disaster's criteria and models $[\mathrm{J}]$. Disaster Prevention and Management , Bradford : 2003,(12)4:305,7.

[5]Qi Jingjing, Chen an. A Study on Emergency Multi-level Phase Mechanism[J], Journal of China Three Gorges University(Humanities\& Social Sciences) ， 2008(30),12:38-39.(In Chinese)

[6]Chen Huanchun, Song Yunfeng. Animal Disease Prevention and Control \& the Development of Animal Husbandry[J], Chinese Poultry Industry, 2009(22). (In Chinese)

[7]Zhang Jianxin, Zhang Linjiang, Liang Songlin. A Study on Pig Epidemic Situation, Trend and Law[J], China Animal Health , 2010.11 31-32(In Chinese)

[8]Merle Jacob, Tomas Hellstrom. Policy understanding of science $[\mathrm{J}]$. Public Trust and the BSE - CJD Crisis Journal of Hazardons Materials. 2000,(78):303-317. 